\title{
Enhancing Label Correlation Feedback in Multi-Label Text Classification via Multi-Task Learning
}

\author{
Ximing Zhang ${ }^{1 * \dagger}$, Qian-Wen Zhang ${ }^{2 *}{\text { Zhao } \text { Yan }^{2} \text {, Ruifang Liu }}^{1}$, Yunbo Cao ${ }^{2}$ \\ ${ }^{1}$ Beijing University of Posts and Telecommunications, Beijing 100876, China \\ ${ }^{2}$ Tencent Cloud Xiaowei, Beijing 100080, China \\ ximingzhang@bupt.edu.cn, cowenzhang@tencent.com, zhaoyan@tencent.com, \\ lrf@bupt.edu.cn,yunbocao@tencent.com
}

\begin{abstract}
In multi-label text classification (MLTC), each given document is associated with a set of correlated labels. To capture label correlations, previous classifier-chain and sequenceto-sequence models transform MLTC to a sequence prediction task. However, they tend to suffer from label order dependency, label combination over-fitting and error propagation problems. To address these problems, we introduce a novel approach with multi-task learning to enhance label correlation feedback. We first utilize a joint embedding (JE) mechanism to obtain the text and label representation simultaneously. In MLTC task, a document-label cross attention (CA) mechanism is adopted to generate a more discriminative document representation. Furthermore, we propose two auxiliary label co-occurrence prediction tasks to enhance label correlation learning: 1) Pairwise Label Co-occurrence Prediction (PLCP), and 2) Conditional Label Co-occurrence Prediction (CLCP). Experimental results on AAPD and RCV1-V2 datasets show that our method outperforms competitive baselines by a large margin. We analyze low-frequency label performance, label dependency, label combination diversity and coverage speed to show the effectiveness of our proposed method on label correlation learning. Our code is available at https://github.com/EiraZhang/LACO.
\end{abstract}

\section{Introduction}

Multi-label text classification (MLTC) is an important natural language processing task with applications in text categorization, information retrieval, web mining, and many other real-world scenarios (Zhang and Zhou, 2014; Liu et al., 2020). In MLTC, each given document is associated with a

\footnotetext{
${ }^{*}$ Equal contribution.

${ }^{\dagger}$ Work done during an internship at Tencent.
}

set of labels which are often related statistically and semantically. Label correlations should be sufficiently utilized to build multi-label classification models with strong generalization performance (Tsoumakas et al., 2009; Gibaja and Ventura, 2015). In particular, learning the dependencies between labels might be helpful in modeling the low-frequency labels, because real-world classification problems tend to exhibit long-tail label distribution, where low-frequency labels are associated with only a few instances and are difficult to learn (Menon et al., 2020).

Previous sequence-to-sequence (Seq2Seq) based methods (Nam et al., 2017; Yang et al., 2018) have been shown to have a powerful ability to capture label correlations with using the current hidden state of the model and the prefix label predictions. However, exposure bias phenomenon (Bengio et al., 2015) may cause the models overfit to the frequent label sequence in training set, thus lead to several problems. First, Seq2Seq-based methods heavily rely on a predefined ordering of labels and perform sensitively to the label order (Vinyals et al.; Yang et al., 2019; Qin et al., 2019). Actually, labels are essentially an order-independent set in the MLTC task. Second, the Seq2Seq-based methods suffer from low generalization ability problem since they tend to overfit the label combinations in the training set and have difficulty to generate the unseen label combination. Third, Seq2Seq-based methods rely on the previous potentially incorrect prediction results. The errors may propagate during the inference stage where true previous target labels are unavailable and are thus replaced by labels generated by the model itself.

To circumvent the potential issues mentioned above, we introduce a multi-task learning based approach that does not rely on Seq2Seq architecture. The approach contains a shared encoder, a MLTC task specific module and a label correla- 
tion enhancing module. In the shared parameter layers, we introduce a joint embedding (JE) mechanism which takes advantage of a transformer-based encoder to obtain document and label representation jointly. Correlations among labels are learned implicitly through the self-attention mechanism, which is different from previous label embedding methods (Wang et al., 2018; Xiao et al., 2019) that treat labels independently. In MLTC task specific module, we generate the label-specific document representation by the document-label cross attention (CA) mechanism, which retains discriminatory information. The shared encoder and the MLTC task specific module form the basic model called LACO, i.e. LAbel COrrelation aware multi-label text classification.

The co-occurrence relationship among labels is one of the important signal that can reflect label correlations explicitly, which can be obtained without additional manual annotation. In label correlation enhancing module, we propose two label co-occurrence prediction tasks, which are jointly trained with the MLTC task. The one is the Pairwise Label Co-occurrence Prediction (PLCP) task for capturing second-order label correlations through the two-by-two combinations to distinguish whether they appear together in the set of relevant labels. The other one is the Conditional Label Co-occurrence Prediction (CLCP) task for capturing high-order label correlations through a given partial relevant label set to predict the relevance of other unknown labels.

We conduct experiments on AAPD and RCV1V2 datasets, and show that our method outperforms competitive baselines by a large margin. Comprehensive experimental results are provided to analysis low-frequency label performance, label dependency, label combination diversity and coverage speed, which are essential to measure the ability of label correlation learning. We highlight our contributions as follows:

1. We propose a novel and effective approach for MLTC, which not only sufficiently learns the features of documents and labels through the joint space, but also reinforces correlations through multi-task design without depending on the label order.

2. We propose two feasible tasks (PLCP and CLCP) to enhance the feedback of label correlations, which is beneficial to help induce the multi-label predictive model with strong generalization performance.

3. We compare our approach with competitive baseline models on two multi-label classification datasets and systematically demonstrate the superiority of the proposed models.

\section{Related Work}

Our work mainly relates to two fields of MLTC task: label correlation learning and document representation learning.

\subsection{Label Correlation Learning}

For MLTC task, a simple but widely used method is binary relevance (BR) (Boutella et al., 2004), which decomposes the MLC task into multiple independent binary classification problem without considering the correlations between labels.

To capture label correlations, label powerset (LP) (Tsoumakas and Katakis, 2007) take MLTC task as a multi-class classification problem by training a classifier on all unique label combinations. Classifier Chains (CC) based method (Read et al., 2011) exploits the chain rule and predictions from the previous classifiers as input. Seq2Seq architectures are proposed to transform MLTC into a label sequence generation problem by encoding input text sequences and decoding labels sequentially (Nam et al., 2017). However, both CC and Seq2Seqbased methods heavily rely on a predefined ordering of labels and perform sensitively to the label order. To tackle the label order dependency problem, various methods have been explored: by sorting heuristically (Yang et al., 2018), by dynamic programming (Liu and Tsang, 2015), by reinforcement learning (Yang et al., 2019), by multi-task learning (Tsai and Lee, 2020; Zhao et al., 2020). Different from these works, our method learns the label correlations through a non-Seq2Seq-based approach without suffering the above mentioned problems.

More recently, researchers have proposed a variety of label correlation modeling methods for MLTC that are not based on Seq2Seq architecture. Wang et al. (2020) propose a multi-label reasoner mechanism that employs multiple rounds of predictions, and relies on predicting multiple rounds of results to ensemble or determine a proper order, which is computationally expensive. CorNetBertXML (Xun et al., 2020) utilizes BERT (Devlin et al., 2019) to obtain the joint representation of 
text and all candidate labels and extra exponential linear units (ELU) at the prediction layer to make use of label correlation knowledge. Different from the above works, we exploit extra label co-occurrence prediction tasks to explicitly model the label correlations in a multi-task framework.

\subsection{Document Representation Learning}

Text representation plays a significant role in text classification tasks. It is crucial to extract essential hand-crafted features for early models (Joachims, 1998). Deep neural network based MLTC models have achieved great success such as CNN (Kurata et al., 2016; Liu et al., 2017), RNN (Liu et al., 2016), CNN-RNN (Chen et al., 2017; Lai et al., 2015), attention mechanism (Yang et al., 2016; You et al., 2018; Adhikari et al., 2019) and etc. (Devlin et al., 2019) is an important turning point in the development of text classification task and it works by generating contextualized word vectors using Transformer. The reason why deep learning methods have become so popular is their ability to learn sophisticated semantic representations from text, which are much richer than hand-crafted features(Guo et al., 2020). However, these methods tend to ignore the semantics of labels while focusing only on the representation of the document.

Recently, label embedding is considered to improve multi-label text classification tasks. (Liu et al., 2017) is the first DNN-based multi-label embedding method that seeks a deep latent space to jointly embed the instances and labels. LEAM (Wang et al., 2018) applies label embedding in text classification, which obtains each label's embedding by its corresponding text descriptions. LSAN (Xiao et al., 2019) makes use of document content and label text to learn the label-specific document representation with the aid of self-attention and label-attention mechanisms. Our work differs from these works in that the goal of our work is to consider not only the relevance between the document and labels but also the correlations between labels.

\section{Methodology}

The framework of LACO is shown in Figure 1. The lower layers are shared across all tasks, while the top layers are task-specific. In this section, we first introduce the standard formal definition of MLTC. After that, we present the detailed technical implementation of LACO.

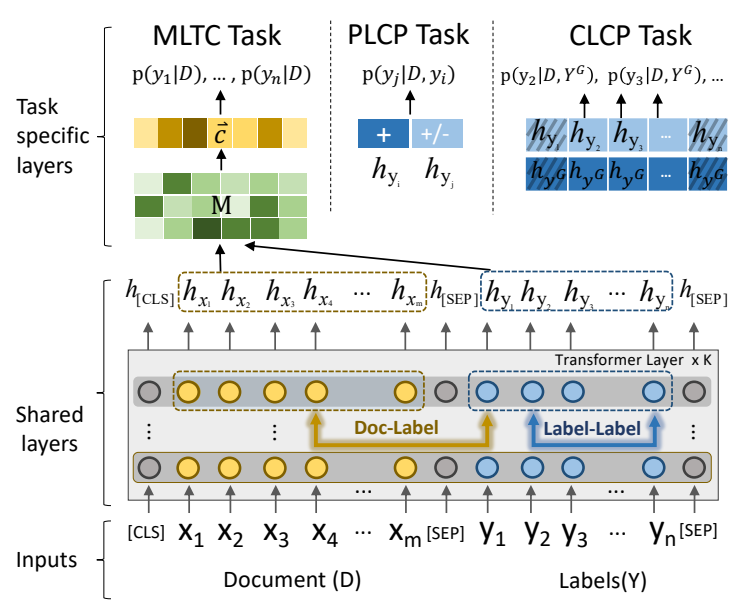

Figure 1: The framework of our proposed approach. Note that the shaded square in the CLCP task is the embedding of given labels, and,+- represent related label and unrelated label respectively.

\subsection{Problem Formulation}

Multi-label task studies the classification problem where each single instance is associated with a set of labels simultaneously. Given a training set $S=\left\{\left(D_{i}, Y_{i}^{+}\right) \mid 1 \leq i \leq N\right\}$ of multi-label text classification data, $D_{i}$ is the text sequence and $Y_{i}^{+}$ is its corresponding labels. Specifically, a text sequence $D$ of length $m$ is composed of word tokens $D=\left\{x_{1}, x_{2}, \ldots, x_{m}\right\}$, and $Y=\left\{y_{1}, y_{2}, \ldots, y_{n}\right\}$ denote the label space consisting of $n$ class labels. The aim of MLTC is to learn a predictive function $f: D \rightarrow 2^{Y}$ to predict the associated label set for the unseen text. For such, the model must optimize a loss function which ensures that the relevant and irrelevant labels of each training text are predicted with minimal misclassification.

\subsection{Document-Label Joint Embedding (JE)}

Following BERT (Devlin et al., 2019), the first token is always the [CLS] token. The output vector corresponding to the [CLS] token aggregates the features of the whole document and can be used for classification. Different from this habitual operation, we propose a novel input structure to directly use label information in constructing the token-level representations.

As shown in Figure 1, the inputs are packed by a sequence pair $(D, Y)$, we separate the text sequence $D$ and the label sequence $Y$ with a special token [SEP]. Note that the label sequence is to concatenate all label tokens. The shared layers map the inputs into a sequence of embedding vectors, one for each token, 
called token-level representations. Formally, let $\left\{[C L S], x_{1}, \ldots, x_{m},[S E P], y_{1}, \ldots, y_{n},[S E P]\right\}$ be the input sequence of the encoder, we obtain the output contextualized token-level representations $\left\{h_{[C L S]}, h_{x_{1}}, \ldots, h_{x_{m}}, h_{[S E P]}, h_{y_{1}}, \ldots, h_{y_{n}}, h_{[S E P]}\right\}$. The input structure is designed to guarantee that words and labels are embedded together in the same space. With the joint embedding mechanism, our model could pay more attention to two facets: 1) The correlations between document and labels Different document have different influences on a specific label, while the same document fragment may affect multiple labels. 2) The correlations among labels. The semantic information of labels is interrelated, and label co-occurrence indicates strong semantic correlations between them.

\subsection{Multi-Label Text Classification}

In this subsection, we introduce the MLTC task specific module, including Document-Label Cross Attention (CA) and Label Predication.

\subsubsection{Document-Label Cross Attention (CA)}

To explicitly model the semantic relationship between each word and label token, we measure the compatibility of label-word pairs via dot product:

$$
M=H_{D} H_{Y}^{T}
$$

where $H_{D}=\left[h_{x_{1}}, \ldots, h_{x_{m}}\right]$ is the text sequence embedding, $H_{Y}=\left[h_{y_{1}}, \ldots, h_{y_{n}}\right]$ is the label sequence embedding and $M \in \mathcal{R}^{m \times n}$. Considering the semantic information among consecutive words, we further generalize $M$ through nonlinearity network. Specifically, for a text fragment of length $2 r+1$ centered at $i$, the local matrix block $M_{i-r ; i+r}$ in $M$ measures the correlation for the label-phrase pairs. To improve the effectiveness of the sparse regularization, we use CNN with ReLU activation in the hidden layers, and perform max-pooling and hyperbolic tangent sequentially in the function $\Omega$ :

$$
\vec{c}=\Omega\left(M_{i-r ; i+r}\right) \cdot H_{D}
$$

Note that the final document representation $\vec{c}$ is generated by aggregation of word representations $H_{D}$, and weighted by the label-specific attention vector $\Omega(\cdot)$.

\subsubsection{Label Predication}

Once having the discriminative document representation, we build the multi-label text classifier via a fully connected layer that captures more finegrained features from different regions of the document:

$$
\vec{p}=\operatorname{sigmoid}\left(W_{1} \vec{c}^{T}+b_{1}\right)
$$

where $W_{1} \in \mathcal{R}^{n \times k}$ and $b_{1} \in \mathcal{R}^{n}$. We use Binary Cross Entropy as the loss function for the multilabel classification problem:

$$
\mathcal{L}_{\text {mlc }}=-\sum_{i=1}^{n}\left[q_{i} \ln p_{i}+\left(1-q_{i}\right) \ln \left(1-p_{i}\right)\right]
$$

where $p_{i}=P\left(y_{i} \mid D\right)$ is the probability of $y_{i}$ predicted by the model, and $q_{i} \in\{0,1\}$ is the categorical information of $y_{i}$. We train the model by minimizing the cross-entropy error.

\subsection{Multi-Task Learning with Label Correlations}

In this subsection, we introduce two auxiliary tasks, Pairwise Label Co-occurrence Prediction (PLCP) and Conditional Label Co-occurrence Prediction (CLCP), to explore the second-order and high-order label relationships, respectively.

\subsubsection{PLCP Task}

Suppose that each document $D$ contains the corresponding label set $Y^{+}$and the uncorresponding label set $Y^{-}$. In order to train the model to understand second-order label relationships, we propose a binarized label-pair prediction task named as PLCP that can be trivially generated from the multi-label classification corpus. The strategy of selecting label pairs for co-occurrence prediction is straightforward. One part is sampled only from $Y^{+}$, which is marked as IsCo-occur, and the other part is sampled from $Y^{+}$and $Y^{-}$, respectively, which is marked as NotCo-occur. To construct the manual training dataset, we empirically set the ratio of IsCo-occur and NotCo-occur to $\gamma$. As Figure 1 shows, we concat the embedding of the two labels $\left[y_{i}, y_{j}\right]$ together as the input features. The additional binary classifier is used to predict whether the state of the two labels is IsCo-occur or NotCooccur. The loss function is as followed:

$$
\mathcal{L}_{\text {plcp }}=-\left[q_{i j} \ln p_{i j}+\left(1-q_{i j}\right) \ln \left(1-p_{i j}\right)\right]
$$

where $p_{i j}=p\left(y_{j} \mid D, y_{i}\right)$ denotes the output probability of the the co-occurrance of the label-pair, and $\mathrm{q}$ is the ground-truth where $q_{i j}=1$ means IsCo-occur and $q_{i j}=0$ means NotCo-occur. 


\subsubsection{CLCP Task}

To further learn the high-order label relationships, we propose the conditional label co-occurrence prediction (CLCP) task. We first randomly pick $s$ labels from $Y^{+}$to form $Y^{G}$, i.e. $Y^{G} \subseteq Y^{+}$, and then predict whether the remaining labels of $Y$ are relevant with them. Specifically, we introduce an additional position vector $E_{Y}=\left[e_{y_{1}}, \ldots, e_{y_{n}}\right]$, where $e_{y_{i}}=0$ indicates that $y_{i}$ at that position is the sampled label, i.e. $y_{i} \in Y^{G}$, and $e_{y_{i}}=1$ indicates $y_{i} \in Y-Y^{G}$. The average of the embedding of the zero-position labels $h_{y^{G}}$ is concatenated to each nonzero-position label embedding as the input features to predict whether each of remaining labels should be co-occurrence when knowing the sampled labels. In Figure 1, $p\left(y_{i} \mid D, Y^{G}\right)$ denotes the probability of $y_{i}$ predicated by the additional sigmoid classifier. The loss for the classification is the sum of binary cross-entropy loss of each nonzero-position:

$$
\mathcal{L}_{\text {clcp }}=-\sum_{i=1}^{n-s}\left[q_{i} \ln p_{i}+\left(1-q_{i}\right) \ln \left(1-p_{i}\right)\right]
$$

where $q_{i} \in\{0,1\}$ is the ground-truth to denote whether the label $y_{i}$ should be co-occurrence with $Y^{G}$, and $p_{i}=p\left(y_{i} \mid D, Y^{G}\right)$ is the output probability of each masked label $y_{i}$.

\subsubsection{Training Objectives}

The same inputs are first fed into the shared layers, then each sub-task module takes the contextualized token-level representations generated by joint embedding and produces a probability distribution for its own target labels. The overall loss can be calculated by:

$$
\mathcal{L}=\mathcal{L}_{m l c}+\alpha \mathcal{L}_{p l c p}+(1-\alpha) \mathcal{L}_{c l c p}
$$

where $\alpha$ is a hyperparameter in $(0,1), \mathcal{L}_{p l c p}$ and $\mathcal{L}_{\text {clcp }}$ are task-specific Cross-Entropy loss for PLCP task and CLCP task, respectively. ${ }^{1}$

\section{Experimental Setup}

\subsection{Datasets}

We validate our proposed model on two multi-label text classification datasets: Arxiv Academic Paper Dataset (AAPD) (Yang et al., 2018) collected 55,840 abstracts of papers in the field of computer

\footnotetext{
${ }^{1}$ We also implement it with three tasks together. Since the two auxiliary tasks have the similar goal, there is no performance gain.
}

\begin{tabular}{lcccc}
\hline Dataset & $|\mathcal{D}|$ & $|Y|$ & $\overline{\left|D_{i}\right|}$ & $\overline{\left|Y_{i}^{+}\right|}$ \\
\hline AAPD & 55,840 & 54 & 163.42 & 2.41 \\
RCV1-V2 & 804,414 & 103 & 123.94 & 3.24 \\
\hline
\end{tabular}

Table 1: Statistics of datasets. Here, $|\mathcal{D}|$ and $|Y|$ denote the total number of documents and labels. $\mid \overline{D_{i} \mid}$ is the average length of all documents. $\overline{\left|Y_{i}^{+}\right|}$means the average number of labels associated with the document.

science, which is organized into 54 related topics. In AAPD dataset, each paper is assigned multiple topics. Reuters Corpus Volume I (RCV1V2) (Lewis et al., 2004) is composed of 804,414 manually categorized newswire stories for research purposes. Each story in the dataset can be assigned multiple topics, and there are 103 topics in total.

Tabel 1 shows statistics of datasets. Each dataset is divided into a training set, a validation set, and a test set. We followed the division of these two datasets by Yang et al. (2018).

\subsection{Evaluation Metrics}

Multi-label classification can be evaluated with a group of metrics, which capture different aspects of the task (Zhang and Zhou, 2014). Following the previous works (Yang et al., 2018; Tsai and Lee, 2020), we adopt hamming loss, Micro/Macro-F1 scores as our main evaluation metrics. Micro/Macro-P and Micro/Macro-R are also reported to assist analysis. A Macro-average will treat all labels equally, whereas a Micro-average will weighted compute each label by its frequency.

\subsection{Comparing Algorithms}

We adopt a various of methods as baselines, which can be divided into two groups according to whether the label correlations are considered.

The first group of approaches do not consider label correlations. Binary Relevance (BR) (Boutella et al., 2004) amounts to independently training one binary classifier (linear SVM) for each label. CNN (Kim, 2014) utilizes multiple convolution kernels to extract text features and then output the probability distribution over the label space. LEAM (Wang et al., 2018) involves label embedding to obtain a more discriminative text representation in text classification. LSAN (Xiao et al., 2019) learns the label-specific text representation with the help of attention mechanisms. We also implement a BERT (Devlin et al., 2019) classifier which first encodes a document into vector space and then outputs the probability for each label independently. 


\begin{tabular}{|c|c|c|c|c|c|c|c|c|c|c|}
\hline \multirow{2}{*}{$\begin{array}{l}\text { Algorithm } \\
\text { BR }^{\dagger} \text { (Boutella et al., 2004) }\end{array}$} & \multicolumn{5}{|c|}{$\begin{array}{c}\text { AAPD dataset } \\
\mathbf{H L} \downarrow|\mathbf{M i}-\mathbf{P} / \mathbf{R} / \mathbf{F} \mathbf{1} \uparrow| \mathbf{M a}-\mathbf{P} / \mathbf{R} / \mathbf{F} \mathbf{1} \uparrow \mid\end{array}$} & $\mathbf{H L} \downarrow$ & $\begin{array}{r}\text { RCV1-V2 dat } \\
\mathbf{M i -} \mathbf{P} / \mathbf{R} / \mathbf{F 1} \uparrow\end{array}$ & tase & $/ \mathbf{R}$ & $\mathrm{F} 1 \uparrow \mid$ \\
\hline & $|0.0316|$ & | 64.4 / 64.8 / 64.6 & - & - & - & $|0.0086|$ & $|90.4 / 81.6 / 85.8|$ & - & - & - \\
\hline $\mathbf{C N N}^{\dagger}(\mathrm{Kim}, 2014)$ & 0.0256 & 84.9 / 54.5 / 66.4 & - & - & 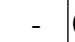 & 0.0089 & $92.2 / 79.8 / 85.5$ & - & - & - \\
\hline LEAM(Wang et al., 2018) & 0.0261 & $76.5 / 59.6 / 67.0$ & $52.4 /$ & $40.3 /$ & 45.6 & 0.0090 & $87.1 / 84.1 / 85.6$ & 69.5 & 5.8 & 67.6 \\
\hline LSAN(Xiao et al., 2019) & 0.0242 & $77.7 / 64.6 / 70.6$ & $67.6 /$ & $47.2 /$ & 53.5 & 0.0075 & $91.3 / 84.1 / 87.5$ & 74.9 & 65.0 & 68.4 \\
\hline BERT(Devlin et al., 2019) & $|0.0224|$ & $|78.6 / 68.7 / 73.4|$ & $68.7 /$ & 521 & 57.2 & 0.0073 & $|92.7 / 83.2 / 87.7|$ & 77.3 & 19 & 66.7 \\
\hline $\mathbf{C C}^{\dagger}($ Read et al., 2011) & 0.0306 & $65.7 / 65.1 / 65.4$ & - & - & - & 0.0087 & $|88.7 / 82.8 / 85.7|$ & - & - & - \\
\hline $\mathbf{S G M}^{\dagger *}$ (Yang et al., 2018) & 0.0251 & $74.6 / 65.9 / 69.9$ & - & - & - & 0.0081 & 88.7 / 85.0 / 86.9 & - & - & - \\
\hline Seq2Set $^{\dagger+}$ (Yang et al., 2019) & 0.0247 & $73.9 / 67.4 / 70.5$ & - & - & - & 0.0073 & $90.0 / 85.8 / 87.9$ & - & - & - \\
\hline OCD $^{\dagger \boldsymbol{H}}$ (Tsai and Lee, 2020) & - & $-\quad-72.0$ & - & & 58.5 & - & $-\quad-$ & - & - & - \\
\hline $\mathbf{M L}^{\prime} \mathbf{R}^{\dagger}$ (Wang et al., 2020) & 0.0248 & $72.6 / 71.8 / 72.2$ & - & - & - & 0.0079 & $89.0 / 85.2 / 87.1$ & - & - & - \\
\hline $\operatorname{Seq} \operatorname{Seq}_{T}^{\mathbf{*}}$ (Nam et al., 2017) & 0.0275 & $69.8 / 68.2 / 69.0$ & $56.2 /$ & $53.7 /$ & 54.0 & 0.0074 & 88.5 / 87.4 / 87.9 & 69.8 & 5.5 & 66.1 \\
\hline SeqTag $_{\text {Bert }}$ & $\mid 0.0238$ & $|74.3 / 71.5 / 72.9|$ & $61.5 /$ & 575 & $58.5 \mid($ & $\mid 0.0073$ & $|90.6 / 84.9 / 87.7|$ & 72 & & 68.7 \\
\hline LACO & 0.0213 & $80.2 / 69.6 / 74$. & 70.4 & $54.0 /$ & 59.1 & $|0.0072|$ & $90.8 / 85.6 / 88.1$ & 75 & & 69.2 \\
\hline $\mathbf{L A C O}+p l c p$ & 0.0212 & $79.5 / 70.8 / 74.9$ & $68.4 /$ & $55.8 /$ & 59.9 & 0.0070 & $90.8 / 86.2 / 88.4$ & & & 69.2 \\
\hline $\mathbf{L A C O}+c l c p$ & 0.0215 & $78.9 / 70.8 / 74.7$ & $71.9 /$ & $56.6 /$ & 61.2 & 0.0070 & 90.6 / 86.4 / 88.5 & 77.6 & & 73.1 \\
\hline
\end{tabular}

Table 2: Predictive performance of each comparing algorithm on two datasets. Hamming Loss (HL), Micro (Mi-) and Marco (Ma-) average Precision (P), Recall (R), F1-Score (F1) are used as evaluation metrics. The $\downarrow$ represents the lower score the better performance, and the $\uparrow$ is the opposite. Models with $\dagger$ denote for its results are quoted from previous papers. Models with os are the Seq2Seq-based models.

The second group of methods consider label correlations. Classifier Chains (CC) (Read et al., 2011) transforms the MLTC problem into a chain of binary classification problems. SGM (Yang et al., 2018) proposes the Seq2Seq model with global embedding mechanism to capture label correlations. Seq2Set (Yang et al., 2019) presents deep reinforcement learning to improve the performance of the Seq2Seq model. We also implement a Seq2Seq baseline with 12-layer transformer, named with Seq2Seq ${ }_{T}$. More recently, OCD (Tsai and Lee, 2020) proposes a framework including one encoder and two decoders for MLTC to alleviate exposure bias. ML-Reasoner (Wang et al., 2020) employs a binary classifier to predict all labels simultaneously and applies a novel iterative reasoning mechanism. Besides, we also provide another strong baseline: SeqTag $_{\text {Bert }}$ transforms multi-label classification task into sequential tagging task, which first obtain embeddings of each label $\left(H_{Y}\right.$ in Sec 3.3) by our shared encoder and then output a probability for each label sequentially by a BiLSTM-CRF model (Huang et al., 2015).

Results of BR, CNN, CC, SGM, Seq2Set, OCD and ML-R are cited in previous papers and results of other baselines are implemented by us. All algorithms follow the same data division.

\subsection{Experimental Setting}

We implement our model in Tensorflow and run on NVIDIA Tesla P40. We fine-tune models on the En- glish base-uncased versions of BERT ${ }^{2}$. The batch size is 32 , and the maximum total input sequence length is 320 . The window size of the additional layer is 10, and we set $\gamma$ as 0.5. We use Adam (Kingma and $\mathrm{Ba}, 2015$ ) with learning rate of 5e-5, and train the models by monitoring Micro-F1 score on the validation set and stopping the training if there is no increase in 50,000 consecutive steps.

\section{Results and Analysis}

In this section, we report the main experimental results of the baseline models and the proposed method on two text datasets. Besides, we analyze the performance on different frequency labels, and further evaluate whether our method effectively learns the label correlations through label-pair confidence distribution learning and label combination prediction. Finally, we give a detailed analysis of the convergence study which demonstrates the generalization ability of our method.

\subsection{Experiment Results}

We report the experimental results of all comparing algorithms on two datasets in Table 2. The first block includes methods without learning label correlations. The second block is the methods considering label correlations, and the third block is our proposed LACO methods. As shown in Table 2, the LACO-based models outperform all

\footnotetext{
${ }^{2}$ https://github.com/google-research/bert
} 


\begin{tabular}{l|cc|cc}
\hline \multirow{2}{*}{ Model } & \multicolumn{2}{|c|}{ AAPD } & \multicolumn{2}{c}{ RCV1-V2 } \\
& HL & Mi-F1 & HL & Mi-F1 \\
\hline BERT & $9.39 \mathrm{e}-09$ & $3.80 \mathrm{e}-10$ & $4.95 \mathrm{e}-04$ & $3.67 \mathrm{e}-08$ \\
SeqTag $_{\text {Bert }}$ & $7.76 \mathrm{e}-16$ & $1.86 \mathrm{e}-07$ & $4.95 \mathrm{e}-04$ & $3.67 \mathrm{e}-08$ \\
\hline
\end{tabular}

Table 3: Statistical analysis results. The P-values of LACO on significant test comparing with the two strong baselines BERT and SeqTag Bert $_{\text {. }}$

baselines by a large margin in the main evaluation metrics. The following observations can be made according to the results:

- Our basic model LACO training only by the MLTC task significantly improves previous results on hamming loss and Micro-F1. Specifically, on the AAPD dataset, comparing to Seq2Set which considers modeling the label correlations, our basic model decreases by $13.8 \%$ on hamming loss and improves by $5.67 \%$ on Micro-F1. Comparing with the label embedding method like LSAN, LACO achieves a reduction of $4.00 \%$ hamming loss score and an improvement of $0.69 \%$ Micro-F1 score on the RCV1-V2 dataset. Also, BERT is still a strong baseline, which shows that obtaining a high-quality discriminative document representation is important for the MLTC task. Here, we train the LACO with 3 random seeds and calculate the mean and the standard deviation. We perform a significant test with LACO and the two strong baselines BERT and SeqTag Bert $_{\text {in Table } 3 .}$ Comparing with the two strong baseline models, all of the P-values of LACO are below the threshold $(p<0.05)$, suggesting that the performance is statistically significant. In addition, we implement Friedman test (Demšar, 2006) for hamming loss and Micro-F1 metrics. The Friedman statistics $F_{F}$ for hamming loss is 7.875 and for Micro-F1 is 6.125 , when the corresponding critical value is 2.8179 (\# comparing algorithms $k=12$, \# datasets $N=2$ ). As a result, the null hypothesis of indistinguishable performance among the compared algorithms is clearly rejected at 0.05 significance level.

- Compared with SGM, Seq2Seq ${ }_{T}$ does not achieve significantly improvements, but SeqTag ${ }_{\text {Bert }}$ shows good performance based on the shared Transformer encoder between document and labels. Notably, the result of SeqTag ${ }_{\text {Bert }}$ on Micro-F1 is comparable to BERT, but the result on Macro-F1 is observably higher. The above illustrates that label correlation information is more important for learning low frequency labels.

\begin{tabular}{l|crr|rrr}
\hline \multirow{2}{*}{ Model } & \multicolumn{3}{|c|}{ AAPD } & \multicolumn{3}{c}{ RCV1-V2 } \\
\hline LACO & HL & Mi-F & Ma-F & HL & Mi-F & Ma-F \\
\hline w/o JE & 0.0213 & 74.5 & 59.1 & 0.0072 & 88.1 & 69.2 \\
w/o CA & 0.0237 & 72.6 & 57.7 & 0.0077 & 87.5 & 68.4 \\
w/o JE \& CA & 0.0220 & 73.5 & 58.4 & 0.0073 & 87.8 & 68.5 \\
\hline
\end{tabular}

Table 4: Ablation over the proposed joint embedding (JE) and cross attention (CA) mechanisms using the LACO model on AAPD and RCV1-V2 datasets.

- As for the results of the multi-task learning methods, the two subtasks introduced by our method have a certain degree of improvement on the main metrics of the two datasets. Specifically, we observe that the PLCP task shows better performance and presents the best score of 74.9 on Micro-F1 for AAPD dataset, while the CLCP task presents the best performance on Micro-F1 for RCV1-V2 dataset as 88.5. Furthermore, the proposed multi-task framework shows great improvements than the basic model LACO on Macro-F1, which demonstrates that the performance on low-frequency labels can be greatly improved through our label correlation guided subtasks. There are more detailed analysis in Section 5.3 and 5.5. Notably, the CLCP task performs better on Marco-F1 by considering the high-order correlations. We also implement the experiment using the losses of three tasks together, while the combination of the two subtasks can not further improve the model performance comparing to $\mathrm{LACO}_{+}$plcp or $\mathrm{LACO}_{+c l c p}$, which we consider is due to the strong relevance between the two tasks.

\subsection{Ablation Study}

In this section, we will demonstrate the effectiveness of two cores of the proposed LACO model, that is a document-label joint embedding (JE) mechanism, and a document-label cross attention (CA) mechanism. Note that, the setting of w/o JE \& CA is equivalent to the BERT baseline in Tabel 2, which encode document only and predict the probability for each label based on [CLS]. In the w/o JE setting, document embedding is encoded by BERT while each label embedding is a learnable random initialized vector. Its label prediction layer is the same with LACO. In the w/o CA setting, document and label embedding are obtained by BERT jointly, and probability for each label is predicted based on [CLS]. Tabel 4 shows that JE and CA are both important to obtain a more discriminative text rep- 


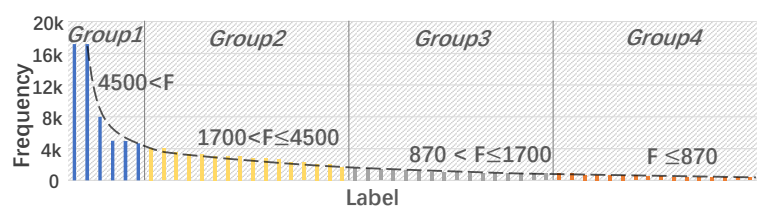

(a) The label distribution of AAPD

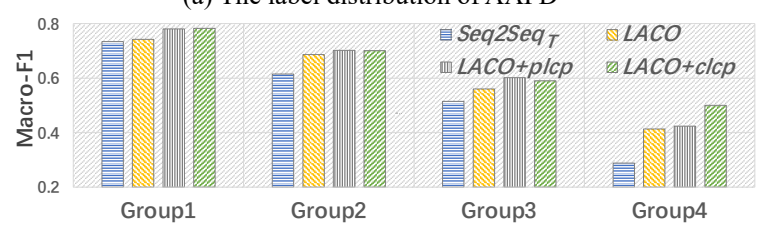

(b)Macro-F1 for the four groups on AAPD

Figure 2: Label classification performance on different frequency distributions. Subfigure(a) shows the label frequency distribution of each label on AAPD training set. Subfigure(b) illustrates the Macro-F1 performance of different methods in the four groups.

resentation. After removing JE and CA mechanism, the performance drops more in the AAPD dataset than RCV1-V2 dataset. We believe that is mainly due to the less of training instance in AAPD, which is more difficult to learn relevant features especially for those low-frequency labels.

\subsection{Low-frequency Label Performance}

Figure 2(a) illustrates the label frequency distribution on AAPD training set, which is a typical big-head-long-tail distribution. We divide all the labels into four groups according to the frequency, the big-head group (Group1), the highfrequency group (Group2), the middle-frequency group (Group3), and the low-frequency group (Group4). As shown in Figure 2(b), we find the performance of all methods decreases with the label frequency of occurrence. The performance gap between Seq2Seq ${ }_{T}$ and LACO based methods increases as the frequency decreases, especially in Group 4, $\mathrm{LACO}_{+c l c p}$ achieves a $74.5 \%$ improvement comparing to the Seq2Seq ${ }_{T}$ model, which demonstrates that the performance on lowfrequency labels can be enhanced by the conditional label co-occurrence prediction task.

\subsection{Label Correlation Analysis}

The co-occurrence relationship between labels is one of the important aspects that can reflect label correlation. In this experiment, we utilize the conditional probability $p\left(y_{b} \mid y_{a}\right)$ between label $y_{a}$ and $y_{b}$ to represent their dependency quantitatively. Furthermore, we calculate the Conditional KullbackLeibler Divergence of $p\left(y_{b} \mid y_{a}\right)$ to measure the "dis-

\begin{tabular}{l|cc|cc}
\hline & \multicolumn{2}{|c|}{$A A P D$} & \multicolumn{2}{c}{$R C V 1-V 2$} \\
Model & train & test & train & test \\
\hline Seq2Seq $_{T}$ & $\mathbf{1 . 2 7}$ & 1.30 & 0.08 & 0.94 \\
SeqTag $_{\text {Bert }}$ & 1.40 & 1.28 & 0.09 & 0.95 \\
LACO & 1.40 & 1.27 & 0.09 & 0.94 \\
LACO $_{+ \text {plcp }}$ & 1.35 & 1.28 & 0.08 & $\mathbf{0 . 7 6}$ \\
LACO $_{+ \text {clcp }}$ & 1.32 & $\mathbf{1 . 1 0}$ & 0.08 & 0.91 \\
\hline
\end{tabular}

Table 5: $K L\left(P^{g} \| P^{p}\right)$ for different models on AAPD and RCV1-V2 datasets. Note that $P^{g}$ is the ground truth distribution of datasets and $P^{p}$ is the model distribution. Smaller scores indicate that two distributions are closer.

tance" between model prediction distribution $\left(P^{p}\right)$ and the ground-truth distribution on training/testing dataset $\left(P^{g}\right)$. The score is calculate as:

$$
\begin{aligned}
K L\left(P^{g} \| P^{p}\right) & =\sum_{y_{a}, y_{b} \in Y}\left(p^{g}\left(y_{b} \mid y_{a}\right) \log \frac{p^{g}\left(y_{b} \mid y_{a}\right)}{p^{p}\left(y_{b} \mid y_{a}\right)}\right. \\
p\left(y_{b} \mid y_{a}\right) & =\#\left(y_{a}, y_{b}\right) / \#\left(y_{a}\right)
\end{aligned}
$$

where \# means the number of the single label or the label combination in the training/testing dataset. The KL-distances on the AAPD and RCV1-V2 datasets are shown in Table 5. On the testing set settings, we can find that LACO has much better fitting ability for the dependency relationship between labels, especially after introducing the co-occurrence relationship prediction task. The Seq2Seq ${ }_{T}$ model achieves the lowest KL distance with training set on both AAPD and RCV1-V2 but achieve larger scores on the test set. This conclusion further proves that the Seq2Seq-based model is prone to over-fitting label pairs during training. It should be emphasized that this KL distance just quantify how much interdependence between label pairs the model have learned, but it cannot directly measure the prediction accuracy of the model.

\subsection{Label Combination Diversity Analysis}

Table 6 shows the number of different predicted label combinations $\left(C_{\text {Test }}\right)$ and subset accuracy $(A c c)$, which is a strict metric that indicates the percentage of samples that have all their labels classified correctly. Seq2Seq ${ }_{T}$ produces fewer kinds of label combinations on the two datasets. As they tend to "remember" label combinations, the generated label sets are most alike, indicating a poor generalization ability to unseen label combinations. Because Seq2Seq $\mathrm{S}_{T}$ is conservative and only generates label combinations it has seen in the training set, it achieves high $A c c$ values, especially on 


\begin{tabular}{l|cc|cc}
\hline & \multicolumn{2}{|c|}{ AAPD } & \multicolumn{2}{c}{ RCV1-V2 } \\
Model & $C_{\text {Test }}$ & Acc & $C_{\text {Test }}$ & Acc \\
\hline Ground Truth & 392 & 1.000 & 278 & 1.000 \\
Seq2Seq $_{T}$ & 214 & 0.392 & 87 & 0.669 \\
OCD $^{\dagger}$ & 302 & 0.403 & - & - \\
SeqTag $_{\text {Bert }}$ & 289 & 0.410 & 187 & 0.637 \\
LACO $^{\text {LACO }}$ & 315 & 0.425 & 241 & 0.642 \\
LACO $_{+ \text {plcp }}$ & 320 & 0.439 & 241 & 0.644 \\
\hline
\end{tabular}

Table 6: Statistics on the number of label combinations. $C_{\text {Test }}$ is the number of different predicted label combinations. Acc is the subset accuracy on the testing set.

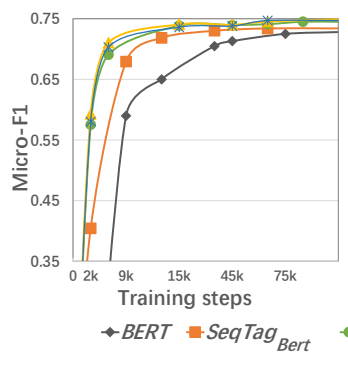

(a) Covergence speed of AAPD

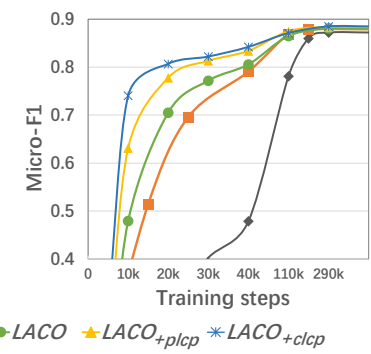

(b) Covergence speed of RCV1-V2
Figure 3: The convergence speed of five BERT-based methods. The $\mathrm{x}$-axis refers to the training steps, and the $\mathrm{y}$-axis refers to the Micro-F1 score performance.

RCV1-V2 dataset. For our models, they produce more diverse label combinations while obtaining good Acc since we do not regard multi-label classification as a sequence generation task that uses a decoder to model the relationship between labels. Instead, we learn the correlations among labels on the encoding side, and the scoring between labels does not interfere with each other, which leads to a higher probability of generating label combinations not seen during training than the Seq2Seq-based models.

\subsection{Coverage Speed}

The convergence speed of five BERT-based models are shown in Figure 3. Our basic model LACO outperforms other BERT-based models in terms of convergence speed, and the proposed multi-task mechanisms are able to enhance LACO to converge much faster. The main reason might be that the feature exchanging through multi-tasks accelerates the model to learn a more robust and common representation.

\section{Conclusions and Future Work}

In this paper, we propose a new method for MLTC based on document-label joint embedding and correlation aware multi-task learning. Experimental results show that our method outperforms competitive baselines by a large margin. Detailed analyses show the effectiveness of our proposed architecture using semantic connections between documentlabel and label-label, which helps to obtain a discriminative text representation. Furthermore, the multi-task framework shows strong capability on low-frequency label predicting and label correlation learning.

Considering the Extreme Multi-label Text Classification that contains an extremely large label set, LACO could be further exploited through scheduled label sampling, hierarchical label embedding strategy, and so on. We hope that further research could get clues from our work.

\section{Acknowledgements}

We would like to thank the ACL reviewers for their valuable comments and Keqing He, Haoyan Liu, Zizhen Wang, Chenyang Liao and Rui Pan for their generous help and discussion.

\section{References}

Ashutosh Adhikari, Achyudh Ram, Raphael Tang, and Jimmy Lin. 2019. Docbert: Bert for document classification. arXiv:1904.08398.

Samy Bengio, Oriol Vinyals, Navdeep Jaitly, and Noam Shazeer. 2015. Scheduled sampling for sequence prediction with recurrent neural networks. In Proceedings of the 28th International Conference on Neural Information Processing Systems-Volume 1, pages 1171-1179.

Matthew R Boutella, Jiebo Luob, Xipeng Shena, and Christopher M Browna. 2004. Learning multi-label scene classification. Pattern Recognition, 37:17571771.

Guibin Chen, Deheng Ye, Zhenchang Xing, Jieshan Chen, and Erik Cambria. 2017. Ensemble application of convolutional and recurrent neural networks for multi-label text categorization. In 2017 international joint conference on neural networks (IJCNN), pages 2377-2383. IEEE.

Janez Demšar. 2006. Statistical comparisons of classifiers over multiple data sets. The Journal of Machine Learning Research, 7:1-30.

Jacob Devlin, Ming-Wei Chang, Kenton Lee, and Kristina Toutanova. 2019. Bert: Pre-training of deep bidirectional transformers for language understanding. In Proceedings of the 2019 Conference of the North American Chapter of the Association for Computational Linguistics: Human Language Technologies, Volume 1 (Long and Short Papers), pages 4171-4186. 
Eva Gibaja and Sebastián Ventura. 2015. A tutorial on multilabel learning. ACM Computing Surveys (CSUR), 47(3):1-38.

Biyang Guo, Songqiao Han, Xiao Han, Hailiang Huang, and Ting Lu. 2020. Label confusion learning to enhance text classification models. arXiv:2012.04987.

Zhiheng Huang, Wei Xu, and Kai Yu. 2015. Bidirectional lstm-crf models for sequence tagging. arXiv:1508.01991.

Thorsten Joachims. 1998. Text categorization with support vector machines: Learning with many relevant features. In European conference on machine learning, pages 137-142. Springer.

Yoon Kim. 2014. Convolutional neural networks for sentence classification. In Proceedings of the 2014 Conference on Empirical Methods in Natural Language Processing (EMNLP), pages 1746-1751.

Diederik P. Kingma and Jimmy Ba. 2015. Adam: A method for stochastic optimization. In 3rd International Conference on Learning Representations, ICLR 2015,San Diego, CA, USA, May 7-9, 2015, Conference Track Proceedings.

Gakuto Kurata, Bing Xiang, and Bowen Zhou. 2016. Improved neural network-based multi-label classification with better initialization leveraging label cooccurrence. In Proceedings of the 2016 Conference of the North American Chapter of the Association for Computational Linguistics: Human Language Technologies, pages 521-526.

Siwei Lai, Liheng Xu, Kang Liu, and Jun Zhao. 2015. Recurrent convolutional neural networks for text classification. In Proceedings of the AAAI Conference on Artificial Intelligence, volume 29.

David D Lewis, Yiming Yang, Tony G Rose, and Fan Li. 2004. Rcv1: A new benchmark collection for text categorization research. Journal of machine learning research, 5(Apr):361-397.

Jingzhou Liu, Wei-Cheng Chang, Yuexin Wu, and Yiming Yang. 2017. Deep learning for extreme multilabel text classification. In Proceedings of the 40th International ACM SIGIR Conference on Research and Development in Information Retrieval, pages 115-124.

Pengfei Liu, Xipeng Qiu, and Xuanjing Huang. 2016. Recurrent neural network for text classification with multi-task learning. In Proceedings of the TwentyFifth International Joint Conference on Artificial Intelligence, pages 2873-2879.

Weiwei Liu, Xiaobo Shen, Haobo Wang, and Ivor W Tsang. 2020. The emerging trends of multi-label learning. arXiv:2011.11197.
Weiwei Liu and Ivor W Tsang. 2015. On the optimality of classifier chain for multi-label classification. In Proceedings of the 28th International Conference on Neural Information Processing Systems-Volume 1 , pages 712-720.

Aditya Krishna Menon, Sadeep Jayasumana, Ankit Singh Rawat, Himanshu Jain, Andreas Veit, and Sanjiv Kumar. 2020. Long-tail learning via logit adjustment. arXiv:2007.07314.

Jinseok Nam, Eneldo Loza Mencía, Hyunwoo J Kim, and Johannes Fürnkranz. 2017. Maximizing subset accuracy with recurrent neural networks in multilabel classification. In Advances in neural information processing systems, pages 5413-5423.

Kechen Qin, Cheng Li, Virgil Pavlu, and Javed Aslam. 2019. Adapting rnn sequence prediction model to multi-label set prediction. In Proceedings of the 2019 Conference of the North American Chapter of the Association for Computational Linguistics: $\mathrm{Hu}$ man Language Technologies, Volume 1 (Long and Short Papers), pages 3181-3190.

Jesse Read, Bernhard Pfahringer, Geoff Holmes, and Eibe Frank. 2011. Classifier chains for multi-label classification. Machine learning, 85(3):333.

Che-Ping Tsai and Hung-Yi Lee. 2020. Order-free learning alleviating exposure bias in multi-label classification. In Proceedings of the AAAI Conference on Artificial Intelligence, volume 34, pages 60386045 .

Grigorios Tsoumakas and Ioannis Katakis. 2007. Multi-label classification: An overview. International Journal of Data Warehousing and Mining (IJDWM), 3(3):1-13.

Grigorios Tsoumakas, Ioannis Katakis, and Ioannis Vlahavas. 2009. Mining multi-label data. In Data mining and knowledge discovery handbook, pages 667-685. Springer.

Oriol Vinyals, Samy Bengio, and Manjunath Kudlur. Order matters: Sequence to sequence for sets.

Guoyin Wang, Chunyuan Li, Wenlin Wang, Yizhe Zhang, Dinghan Shen, Xinyuan Zhang, Ricardo Henao, and Lawrence Carin. 2018. Joint embedding of words and labels for text classification. In Proceedings of the 56th Annual Meeting of the Association for Computational Linguistics (Volume 1: Long Papers), pages 2321-2331.

Ran Wang, Robert Ridley, Weiguang Qu, Xinyu Dai, et al. 2020. A novel reasoning mechanism for multilabel text classification. Information Processing \& Management, 58(2):102441.

Lin Xiao, Xin Huang, Boli Chen, and Liping Jing. 2019. Label-specific document representation for multi-label text classification. In Proceedings of the 2019 Conference on Empirical Methods in Natural Language Processing and the 9th International 
Joint Conference on Natural Language Processing (EMNLP-IJCNLP), pages 466-475.

Guangxu Xun, Kishlay Jha, Jianhui Sun, and Aidong Zhang. 2020. Correlation networks for extreme multi-label text classification. In Proceedings of the 26th ACM SIGKDD International Conference on Knowledge Discovery \& Data Mining, pages 1074 1082.

Pengcheng Yang, Fuli Luo, Shuming Ma, Junyang Lin, and Xu Sun. 2019. A deep reinforced sequence-toset model for multi-label classification. In Proceedings of the 57th Annual Meeting of the Association for Computational Linguistics, pages 5252-5258.

Pengcheng Yang, Xu Sun, Wei Li, Shuming Ma, Wei Wu, and Houfeng Wang. 2018. Sgm: sequence generation model for multi-label classification. In Proceedings of the 27th International Conference on Computational Linguistics, page 3915-3926.

Zichao Yang, Diyi Yang, Chris Dyer, Xiaodong He, Alex Smola, and Eduard Hovy. 2016. Hierarchical attention networks for document classification. In Proceedings of the 2016 conference of the North American chapter of the association for computational linguistics: human language technologies, pages 1480-1489.

Ronghui You, Zihan Zhang, Ziye Wang, Suyang Dai, Hiroshi Mamitsuka, and Shanfeng Zhu. 2018. Attentionxml: Label tree-based attention-aware deep model for high-performance extreme multi-label text classification. arXiv:1811.01727.

Min-Ling Zhang and Zhi-Hua Zhou. 2014. A review on multi-label learning algorithms. IEEE transactions on knowledge and data engineering, 26(8):1819-1837.

Wei Zhao, Hui Gao, Shuhui Chen, and Nan Wang. 2020. Generative multi-task learning for text classification. IEEE Access, 8:86380-86387. 